\title{
Research on Three Pillars of Human Resources Management -Taking Huawei as an Example
}

\author{
Xingze $\mathrm{Wu}^{1}$, Dicheng $\mathrm{Wang}^{2}$, Chun Huang ${ }^{1}$, Linsheng Xiao ${ }^{1, *}$ \\ ${ }^{1}$ Beijing Institute of Technology, Zhu Hai, Zhu Hai, Guangdong Province, China \\ ${ }^{2}$ Room 301B, Yaohuazhang building, no. 6023, Shennan avenue, Shatou street, Futian district, Shenzhen, China \\ *Corresponding author. Email: staresque@126.com
}

\begin{abstract}
Researches on three pillars of HRM are at the forefront of HRM. This article summarizes researches on three pillars, analyzes the case of Huawei's three pillars of HRM, and explores the application of the three pillars in the enterprise to provide references for related research and applications.
\end{abstract}

Keywords: Three pillars, Frontiers, HRM

\section{DEFINITION OF THREE PILLARS OF HUMAN RESOURCE MANAGEMENT (HRM)}

Professor Dave Ulrich of the United States re-divided the functions of HRM into three directions: SSC (Shared Service Center), HRBP (Human Resources Business Partner) and COE (Center Of Expert), collectively referred to three-pillar model of human resources. Among them, SSC refers to the unified processing and sharing of all basic administrative work related to HRM in each business unit of the enterprise; HRBP refers to the bridge between human resources and business managers. HRBP should not only be familiar with various areas of HR, but also understand business needs, and assist business managers to better use various HRM systems and tools to manage employees; $\mathrm{COE}$ is mainly responsible for providing professional consulting on human resources for business units. In enterprises, three pillars are coordinated with each other. COE designs policies around the strategic goals of the organization, which are decomposed by HRBP and then implemented by SSC [1].

Three-pillar model of HRM is based on innovations in the organization and management of human resources. Human resources system supported by three-pillars model originates from strategy of company and serves business of company. Its core idea is to rebuild the organization's capabilities to help HRM to create more value.

\section{COMPARISON OF THREE PILLARS OF HRM AND TRADITIONAL HRM}

\subsection{Traditional HRM Model}

Organizational structure of traditional HRM is divided according to professional functions, such as planning, recruitment, training, salary and benefits, performance, and employee relations etc. Although the responsibilities of each position are clear, HRM often just serve for leaders, rather than employees of enterprise. Policy of HRM lacks the pertinence and flexibility to the business. Business department feels more control rather than support. HRM focuses a lot of time on daily affairs, thus cannot effectively provide targeted assistance to meet actual business needs.

\subsection{Three Pillars of HRM}

Three-pillar model is a new type of HRM. For improving efficiency and effectiveness, HRM work is divided into three types. The first type is Human Resource Sharing Service Center (SSC)-which is responsible for providing various daily affairs services. The second type is human resources business partners (HRBP) which provides HRM consulting service to business departments. HRBP is key to ensure that HRM service can meet business needs. The third type is Center Of Expert(COE), which is responsible for formulating corporate human resources strategic plans, formulating various work policies, systems, processes, and developing human resource products. The ultimate goal of three pillars is to break the past function-centric management model and return to business, thereby creating greater value [2].

\section{ADVANTAGES AND LIMITATIONS OF THE THREE PILLARS OF HRM}

Practice shows that the three-pillar model is more suitable for large-scale enterprises, but not for small enterprises. 


\subsection{Advantage}

\subsubsection{Generate scale effects, reduce costs and improve efficiency}

The three-pillar model centralizes and handles repetitive transactional work, which produce an integration scale effect, thereby reducing unit costs and improving efficiency.

\subsubsection{Effective separation of HRM and service functions}

Transactional service functions that can be implemented in a process are handled by the SSC, leaving the highly complex technical functions to $\mathrm{COE}$, while HRBP only needs to focus on dynamic demand changes of business department, provide corresponding solutions, and finally realize the transition from function orientation to business orientation.

\subsection{Limitations}

\subsubsection{Insufficient ability of HRBP itself}

The responsibilities of the HRBP in three pillars are not very clear. HRBP persons can be divided into two categories: HRBP converted from human resources department to business department, and HRBP who has been engaged in departmental business work. For HRBP with a HRM background, they can be better qualified for the department's human resources development due to the advantages of HRM expertise, but they need to improve knowledge and skills of the company's business departments. HRBP of the department's business work knows situations of the business department very well, but it lacks expertise in human resources and may encounter professional bottlenecks.

\subsubsection{Issues on cooperation of business department}

HRBP can become a partner of department, but it is still an issue that whether department can truly accept, cooperate and support HRBP. In the initial stage of HRBP entering the department, it may raise problems in communication and coordination with business department. Besides, whether HRBP is given sufficient authority to carry out work is essential.

\subsubsection{Scale effects is needed}

For small-scale companies, three-pillar model does not have the advantage of scale effect. On the contrary, the workload of COE and HRBP is difficult to reach saturation. Because the work system is too detailed, it generates a lot of communication costs [2].

\subsubsection{COE construction is difficult}

At present, most of the internal HRM work is still at the transactional stage. Due to lack of professional ability, it cannot play the role of expert well. External experts also have certain problems. Due to lack of understanding of the corporate sector, they may not be qualified as experts in practice. Furthermore, experts are mainly based on the company's strategy to formulate human resources policies, institutional systems, etc. If the company still has thought of official standard, experts will not fully create their value.

\subsubsection{The construction of HRBP team is the most difficult}

HRBP business partners have higher requirements on the knowledge system such as professional knowledge and business sensitivity of HR workers, but in reality such HR workers are more scarce than experts. Thus many partners can only play the role of buddies in the end.

\section{CASE ANALYSIS OF THREE PILLARS OF HUAWEI COMPANY}

Huawei's three-pillar system of HRM consists of three parts: a shared service center, an expert center, and business partners. Huawei's SSC (shared service center) handles the daily affairs and administrative services of HRM within the enterprise. According to the company's strategic development goals, it provides service optimization solutions from within the company, optimizes the flow of transactional work. Supervision and control the current external policy trends and the efficiency of internal affairs. Huawei's COE (Human Resources Expert) is mainly responsible for innovation and formulation of HRM policies and institutional processes, providing technical cooperation for HRBP, and working with HRBP to promote HRM systems and programs. Provide suggestions and plans to the company's strategic leadership based on the feedback from the implementation of the system. 


\subsection{Three Pillars of HRM Impact on Each Other}

Huawei's three pillars perform their respective functions. SSC focuses on the company's daily affairs and efficiency improvement. HRBP conducts business unit cooperation and problem feedback. COE formulates solutions to the problems reported by HRBP, innovates and optimizes corporate human resources policies. On the other hand, three pillars impact on each other. HRBP collects and summarizes the problems existing in the business department, and then feeds back to COE. COE formulates policies and solutions and delivers them to the SSC for implementation. SSC will promote the improvement of HRBP management and customer relationships.

\subsection{Diversified Roles of HRBP}

Huawei's HRBP plays diversified roles in business departments, paying attention to needs and problems of various business departments, customizing different HRM solutions, and feeding back key issues to the COE. Departments at different stages have different solutions. In the short-established departments, HRBP aims at the departmental human resources system construction and personnel planning, promotes departmental cohesion and development planning, and enables the rapid development of business departments; in departments with poor performance, HRBP seeks departmental solutions and passes Professional skills and means such as employee relationship management, improve the cohesion and business capabilities of the departments; for departments with outstanding performance, HRBP puts forward suggestions based on the department's strategic planning and future development, and promotes the internal members of the department to higher goals [3].

\subsection{Integrate Corporate Culture into Three Pillars}

Huawei successfully integrated construction of corporate culture into three pillars. The basis of combining the HRBP system with department development and operation is the combination of corporate culture. Core of building HRBP in Huawei is construction of corporate culture. The customer-centric corporate culture enables HRBP to clearly recognize its positioning, provide business support for the Department, improve the process system, improve the efficiency of affairs, and provide human resources services for business department.

\subsection{Pay Attention to Continuous Improvement of Three Pillars}

Huawei managers attach great importance to the quality and skills of HRBP and expert personnel. They have strict control over the selection of HRBP and human resources experts. A competency model selection system ensures the quality of HR experts and HRBP personnel.

\section{SUGGESTIONS}

For the common problems in three pillars of HRM., the following suggestions are proposed.

\subsection{Establish a Strong Organization for Unified Leadership}

Building a HRBP leadership group is a key point in building HRBP. It is directly led by the senior management of human resources and formulates the implementation plan of each module.

\subsection{Avoid Inappropriate Transformation Methods}

There are problems of inappropriate transformation methods of three pillars, which are specifically manifested as follows: (1) Too hastily. Establishment of three pillars is a gradual process. It needs to be a transition between existing functions and ideal functions. (2) Formalization. Three pillars are business-driven needs that require comprehensive and detailed design in terms of responsibilities, rights, benefits, and people. (3) Fragmented Most organizations fail to conduct a systematic analysis from perspective of the value chain of how HRM drives business development, resulting in design goals, paths, principles, methods, and matching management mechanisms that are too fragmented [4].

\subsection{Determine the Transition Environment and Influencing Factors}

Not all organizations are suitable for three pillars. The transformation of HRM to HRBP mode can be carried out when organization develops on a large scale and across regions.

\subsection{Focus on HRM Ability Training Needed by HRBP}

During construction of HRBP, there are certain requirements for the level of HR management: HRBP not only needs to build on HRM skills, but also has certain achievements in corporate culture, business development, organizational influence, and information acquisition capabilities [5]. COE must emphasize strategic thinking, organizational innovation, system optimization and reform, and other basic capabilities. SSC's work with HRBP and 
COE requires HRM to have a certain level of service awareness and execution to meet the basic needs of three pillars.

\subsection{Three Pillars Cooperate with Each Other and Cycle}

In construction of three pillars of HRM, attention should be paid to the mutual cooperation of three pillars and continuous improvement.

\subsection{Integrate Corporate Culture into Three Pillars}

Corporate culture is the foundation of three pillar construction. On the other hand, three pillars reflect the corporate culture. We should pay attention to integrate corporate culture into three pillars.

\section{CONCLUSION}

This paper first defines the three pillars of human resource management, compares the three pillars of human resource management with traditional human resource management, then discusses the advantages and limitations of the three pillars of human resource management, and analyzes the application case of three pillars of human resource management in Huawei Company.Finally, we put forward practical suggestions on the use of the three pillars of human resource management.

\section{REFERENCES}

[1] M. Yanting Li and Lei Tan, Business changes in the Internet age, New World Press, Beijing, 2016.06.

[2] J. Chunhua Wang, Exploring the Intermediate Mode of Traditional HRM and Three Pillars Mode, Human Resource Development, 2017.

[3] J. Ailin Li and Jingxi He, The transformation of HRMfunctions from the perspective of sharing economy: Huawei HRBP for the case Human Resource Development in China. 2016. No. 24: 54-57.

[4] J. Zhenhua Bai, How to transform traditional HRM to HRBP, New Business Week, 2019.15.

[5] J. Minglei Ge, Exploratory Research on Project HRBP Reserve Talents Cultivation-Taking Huawei as an Example, Human Resource Development in China2015, No. 18. 